\title{
Bilateral Orbitofrontal Repetitive Transcranial Magnetic Stimulation in Frontal Lobe Epilepsy: A Case Report
}

\author{
Georgios Mikellides $^{a}$ b $\quad$ Panayiota Michael ${ }^{b} \quad$ Angelos Gregoriou $^{c}$ \\ Teresa Schuhmann ${ }^{a} \quad$ Alexander T. Sack ${ }^{a, d}$ \\ aFaculty of Psychology and Neuroscience, Maastricht University, Maastricht, The \\ Netherlands; ${ }^{b}$ Cyprus rTMS Centre, Larnaca, Cyprus; ${ }^{c}$ Consultant Neurologist and \\ Epileptologist, Aretaeio Private Hospital, Nicosia, Cyprus; ${ }^{\mathrm{d} D e p a r t m e n t}$ of Psychiatry and \\ Neuropsychology, School for Mental Health and Neuroscience (MHeNs), Brain+Nerve \\ Centre, Maastricht University Medical Centre+ (MUMC+), Maastricht, The Netherlands
}

Keywords

Epilepsy · Drug-resistant epilepsy · Orbitofrontal cortex · Repetitive transcranial magnetic stimulation

\begin{abstract}
Epilepsy is a common and severe neurological disorder affecting millions of people worldwide. Nowadays, antiseizure medications (ASMs) are the main treatment for most epilepsy patients, although many of them do not respond to ASMs and suffer from drug-resistant epilepsy (DRE). Alternative and novel treatment methods have been offered nowadays, showing promising results for the treatment of DRE. Repetitive transcranial magnetic stimulation (rTMS) is a noninvasive method that has become increasingly popular in the last decades. This article reports a patient with frontal lobe epilepsy. We aimed to investigate whether bilateral orbitofrontal (OFC) low-frequency rTMS (LF-rTMS) is feasible and tolerable, safe, and potentially clinically effective in treating epileptic seizures. The patient's satisfaction with rTMS therapy was self-reported to be high, as rTMS helped in reducing the frequency of the focal attacks and completely abolished the preceding feeling of fear and panic. Therefore, bilateral OFC rTMS treatment can be well tolerated in patients with frontal epilepsy although the findings of the present case report with regard to clinical efficacy warrant further investigation.
\end{abstract}




\section{Introduction}

Epilepsy is a common and severe neurological disorder affecting approximately 50 million people worldwide [1]. According to the International League Against Epilepsy (ILAE) and the International Bureau for Epilepsy (IBE), epilepsy can be defined as "a disorder of the brain characterized by an enduring predisposition to generate epileptic seizures," with at least a history of one seizure, and by the "neurobiological, cognitive, psychological, and social consequences of this condition" [2]. Antiseizure medications (ASMs) are the main and often effective treatments for most epilepsy patients. Nonetheless, approximately $25 \%$ of epilepsy patients suffer from drug-resistant epilepsy (DRE), not adequately responding to any available combination of ASMs [3]. A recent systematic review and meta-analysis revealed that the cumulative incidence of DRE was $14.6 \%$ in adult/mixed-age studies as well as that the prevalence of DRE was $13.7 \%$ in community-based populations and $36.3 \%$ in clinic-based populations [4].

Nonpharmacological treatment options are actively explored to address the need for treatment alternatives in DRE patients. Recently, immunomodulatory therapies in epilepsy have been introduced, such as corticosteroids, intravenous immunoglobulins, plasmapheresis, and steroid-sparing drugs such as azathioprine [5]. Other treatment options include dietary changes [6], brain surgery [7], and also brain stimulation, including deep brain stimulation [8] and neuromodulation techniques [9]. Regarding neuromodulation techniques, several noninvasive stimulation therapies are currently available for patients with epilepsy such as transcranial electric stimulation [10], transcranial direct current stimulation [11], and transcranial magnetic stimulation (TMS) [12]. Repetitive TMS (rTMS) uses time-varying electromagnetic pulses applied transcranially, through the intact scalp, via an insulated electromagnetic coil placed over a specific area of the brain in order to modulate the underlying cortical excitability [13]. In the past 20 years, rTMS has shown to be clinically effective in treating various neurological and psychiatric disorders such as neuropathic pain or major depressive disorder [14]. At the same time, rTMS has shown to be very well tolerated with very few to no side effects [15]. The ability of rTMS to modulate cortical excitability also for longer periods outlasting the stimulation itself has shown to depend on specific stimulation parameters such as intensity, frequency, number of sessions, and duration of stimulation [13]. Regarding stimulation frequency, low-frequency rTMS (LF-rTMS) $(<1 \mathrm{~Hz}$ ) generally decreases cortical excitability, whereas high-frequency rTMS ( $>5 \mathrm{~Hz}$ ) generally increases cortical excitability, triggering longer-lasting neuroplasticity processes resembling long-term depression or long-term potentiation, respectively [13]. The most serious adverse event of rTMS is the potential induction of seizures. However, seizure induction occurs very rarely [16, 17]. A recently published article regarding safety recommendations for the use of TMS in healthy volunteers and patient populations highlighted factors that are increasing the risk for TMS-provoked seizures. These include the presence of neuropsychiatric diseases associated with structural cerebral damage (e.g., stroke), some medical conditions (e.g., metabolic abnormalities), and general factors like sleep deprivation and increased alcohol consumption [15]. In 2019, Lerner et al. [16] presented the results of a survey among TMS laboratories and clinics, conducted between 2012 and 2016, and reported that the statistical likelihood of inducing a seizure with rTMS is extremely low for participants without such risk factors ( $<1$ seizure per 60,000 sessions).

A small but growing number of studies also investigated the potential of LF-rTMS in treating epileptic patients $[18,19]$. Here, we describe a case report of a patient suffering from frontal lobe epilepsy who underwent LF-rTMS over both orbitofrontal cortices. The patient was followed over a course of 30 sessions to investigate whether bilateral prefrontal

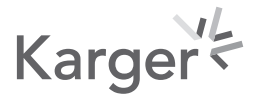



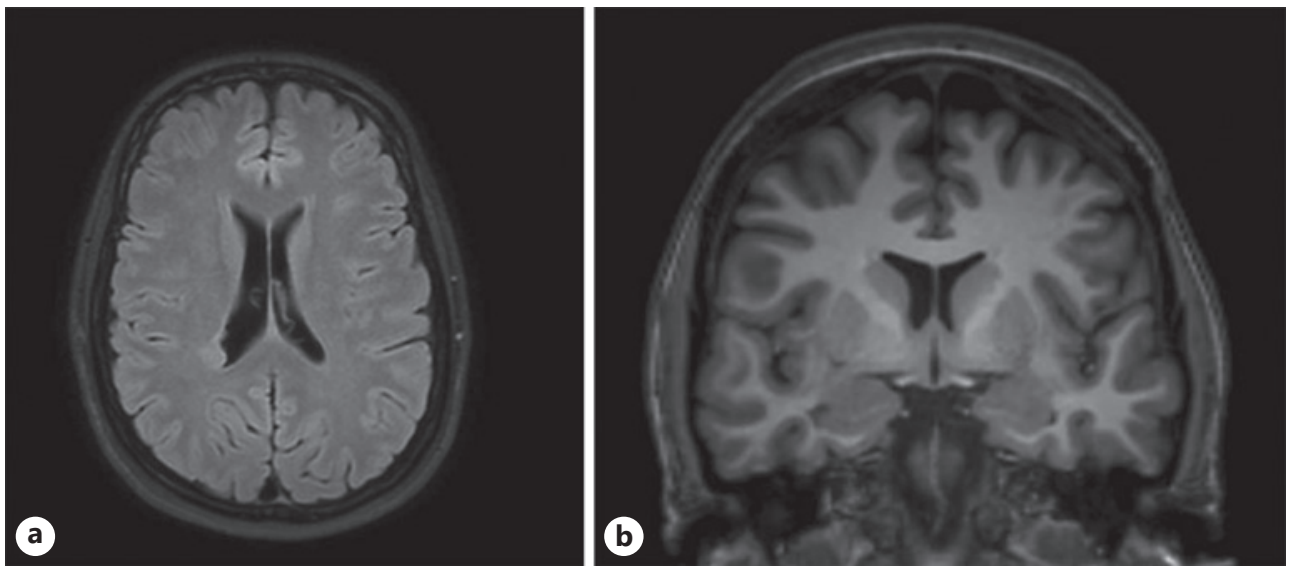

Fig. 1. MRI scans: bilateral subependymal periventricular heterotopia.

LF-rTMS is feasible and tolerable, safe, and potentially clinically effective in treating epileptic seizures.

\section{Case Report/Case Presentation}

\section{Participant}

In this case report, a 28-year-old female patient presented suffering from frontal lobe epilepsy. She first experienced a focal to bilateral tonic-clonic seizure at the age of 14 followed by a similar event at the age of 18. MRI was performed after the first seizure revealing bilateral subependymal periventricular heterotopia, a neuronal migration disorder notoriously resulting in DRE in the majority of cases (Fig. 1). There was also an impression of mild degree of cortical dysplasia in the insular cortex on both sides, slightly more prominent in the left side. At the age of 19, she experienced 4-5 focal to bilateral tonic-clonic seizures per week. After being treated with various combinations of ASMs, the frequency of generalized seizures was reduced to 1 per year for the following 3 years (the focal events continued). By the age of 23 , the generalized seizures were well controlled by medication, but she continued to experience daily focal seizures (1-3 times per day) characterized by episodes of a sudden onset of fear of impending doom associated with tachycardia and sweating, followed by a "strange" sensation in the right face spreading to the right hemitongue, occasionally accompanied by right hand numbness, lasting for less maximally $30 \mathrm{~s}$ without any loss of consciousness. We assume that these episodes involved several structures including the amygdala and the insula (as part of the central autonomic network), spreading to involve also the postcentral gyrus and somatosensory cortex causing the sensory disturbances.

\section{rTMS Treatment}

The patient has been on treatment with different ASMs starting at the onset of the first seizures. Some of the medications used were topiramate, levetiracetam, clobazam, lamotrigine, and gabapentin. The doses of her medications were not standard, as dosing had been changed frequently in an attempt to better control the attacks. Prior to rTMS treatment, the patient was on lamotrigine $375 \mathrm{mg} /$ day. Whilst undergoing rTMS treatment, the patient continued taking levetiracetam 2,500 mg/day and started reducing the dose of lamotrigine by $25 \mathrm{mg}$ every fortnight in an attempt to achieve monotherapy as she aimed to become pregnant in

\section{Karger'}




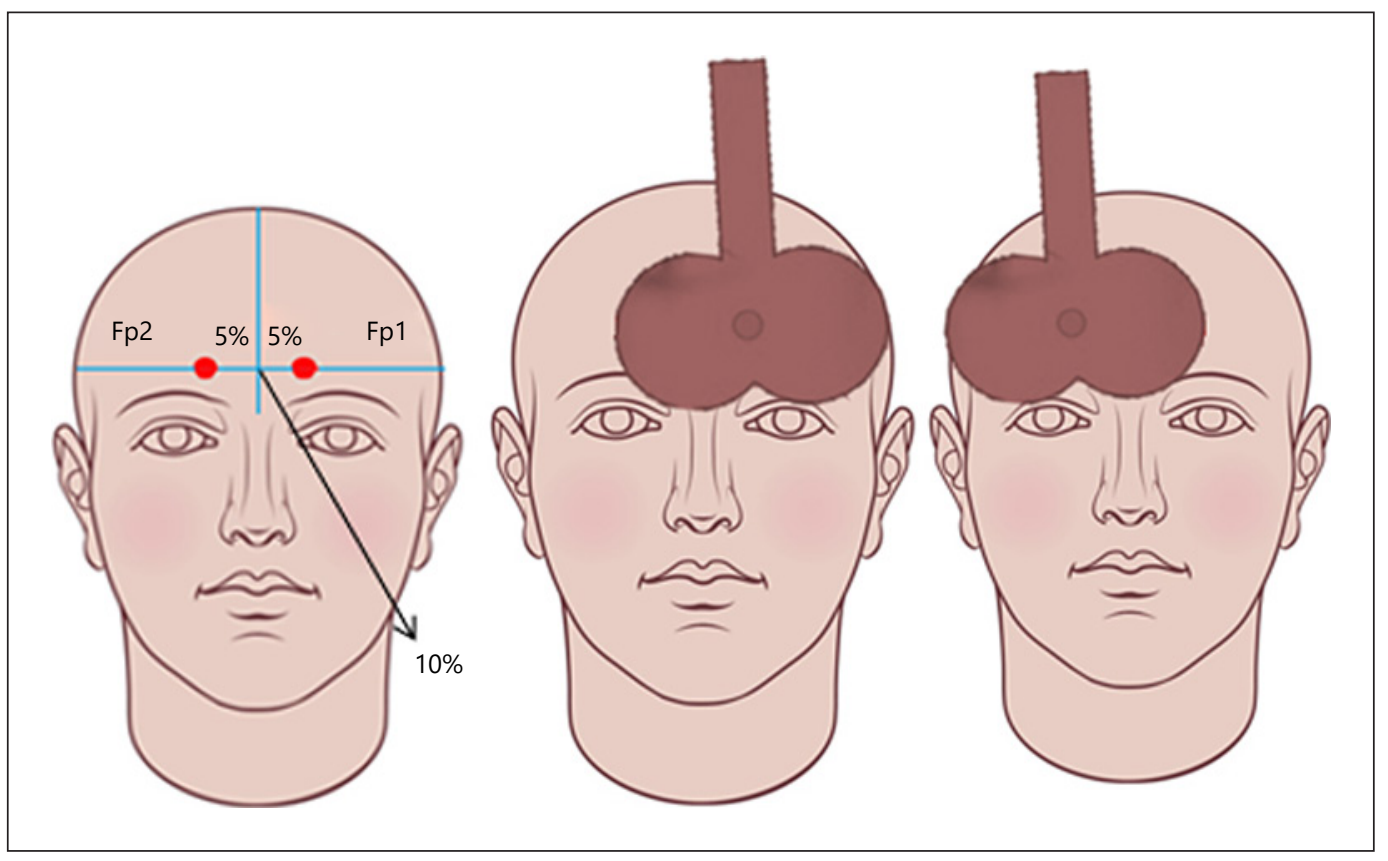

Fig. 2. Coil placement: a stimulation areas: to localize Fp1 and Fp2 within the 10-20 EEG system, we measured $10 \%$ of the nasion to inion distance along the midline (site FPz in the 10-20 EEG), followed by measuring 5\% of the head circumference on the left and right of Fpz (Fp1 and Fp2). b Coil orientation at the left side. c Coil orientation at the right side.

the near future. She also started taking vitamin D 50,000 IU once every month and a daily dose of folic acid $5 \mathrm{mg}$, B complex, and iron supplements.

Written informed consent for the rTMS treatment was obtained from the patient. In the first session, the patient's resting motor threshold (rMT) was determined over the left primary motor cortex. rMT is the amount of machine output (intensity) required to elicit a motorevoked potential in at least $50 \%$ of all attempts [20]. The patient underwent rTMS using the MagPro X100 stimulator (MagVenture, Farum, Denmark), with a figure-eight coil (MC-B70). The TMS coil was placed over the left and right orbitofrontal cortex sequentially (left side for 8, $23 \mathrm{~min}$ and right side for 8, $23 \mathrm{~min}$ ), positioned over the Fp1 and Fp2 EEG sites according to the 10-20 EEG system (Fig. 2). Each rTMS session consisted of 12 trains of 42 pulses admin-

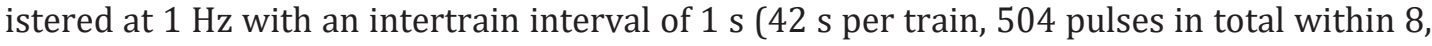
$23 \mathrm{~min}$ ). Stimulation intensity was set at $100 \%$ of the rMT for the first day of treatment, $110 \%$ of rMT for the second day, and then at $120 \%$ of rMT for the remaining treatment days.

The patient received a total of 30 rTMS sessions during the first 5-week period (the patient came to the clinic 3 days per week, and on each visit, she received 2 rTMS sessions, left and right OFC, with a 30-min break between sessions). After the completion of the 30 sessions, the patient continued with 10 maintenance sessions (once weekly for 1 month, once fortnightly for 1 month, and once monthly for the following 4 months as maintenance). To the best of our knowledge, this is the first time that this form of sequential bilateral OFC rTMS was used in a patient with epilepsy and was personalized to the patient's specific epileptic seizures as she received low-frequency inhibition of both orbitofrontal cortices. The orbitofrontal cortex was chosen as a target due to its accessibility as well as due to its significant connectivity with the amygdala and the rest of the central epileptic network [21,22]. Bilateral stimulation was chosen due to the bilateral nature of the patient's neuronal migration disorder despite the unilaterality of the patient's symptoms during most seizures. 


\section{Results}

The patient reported a quick reduction in seizure frequency after the first 2 weeks of treatment (seizure frequency dropped from 1-3 per day to 2-3 per week). After 30 sessions, the patient reported further frequency reduction (seizures appeared only 1-2 per week), and also reported that while she was still feeling an aura of fear on a daily basis, this was now not automatically followed by the above-reported sequence of semiology anymore. During the maintenance period, seizures remained on low frequency with 1-2 seizures per week, including a further reduction in duration and intensity and with the absence of any feeling of fear or panic.

Toward the end of the maintenance period, she started increasing the dose of levetiracetam to $3,000 \mathrm{mg} /$ day and reducing lamotrigine to $100 \mathrm{mg} /$ day in an attempt to achieve monotherapy as mentioned above. Unfortunately, after this reduction of lamotrigine, she experienced 3 focal to bilateral tonic-clonic seizures within 1 month resembling the seizures she had experienced at younger age. Consequently, the dose of lamotrigine was reinstated at $200 \mathrm{mg}$ /day with a return to a better control of seizures.

Overall, the patient's satisfaction with rTMS therapy was self-reported to be high $(70 \%$ satisfied with treatment outcome), as rTMS helped in reducing the frequency of the focal attacks and completely abolished the preceding feeling of fear and panic. It also allowed her to reduce the dose of her ASMs, which resulted in reduction in at least some of the side effects caused by ASMs. No adverse events of rTMS were reported during the whole treatment period.

\section{Discussion}

In this case report, we demonstrated that sequentially applied bilateral OFC rTMS is a safe and well-tolerated treatment for DRE resulting from bilateral subependymal heterotopia. We also showed that rTMS did reduce the frequency, duration, and intensity of the patient's seizures.

Although the induction of seizures is reported as the most serious side effect of rTMS, the actual occurrence of seizures as a consequence of rTMS can be considered as extremely rare with a probability of $<1$ in 60,000 sessions [15-17]. Several studies aimed to investigate the effectiveness of TMS in epilepsy using different stimulation parameters and positions (Table 1). The present patient underwent LF-rTMS, as LF-rTMS could help inhibit the brain overactivity, which potentially will cause a new neuroadaptation to the brain circuits, preventing them from overfiring and causing epileptic seizures. This case report adds to the existing literature regarding the safety and effectiveness of LF-rTMS in the reduction of seizure frequency in patients with medically refractory epilepsies [23, 24]. A recent metaanalysis suggested that LF-rTMS may indeed be an effective therapy for patients with DRE based on its ability to reduce cortical excitability and consequently reduce seizure frequency and interictal epileptiform discharges [18]. Similarly, according to a randomized controlled trial, LF-rTMS can produce antiepileptic effects in patients with refractory focal seizures [19]. rTMS has here and in other studies indicated to be potentially effective without causing rTMStriggered seizures. It may therefore be considered as an additional therapeutic tool in the treatment of DRE. In addition, our results showed that panic attacks prior to the onset of an episode may be strongly reduced during rTMS treatment. It has been clearly shown in the past that LF-rTMS to the right dorsolateral prefrontal cortex may result in clinical improvement of panic disorder and reduce the ipsilateral motor cortex excitability [25]. Of course, it must be clearly stated here that this case report is not suited to draw any scientifically strong conclusion with regard to the clinical efficacy of OFC rTMS in treating frontal lobe epilepsy

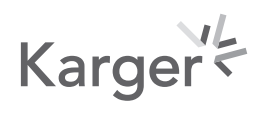


due to the complete absence of a control or placebo condition as well as to the unique case reported here mainly in descriptive and qualitative terms.

Nonetheless, this case report may be the first to mention the safe and feasible application of sequential rTMS to the bilateral orbitofrontal cortex in a patient with epilepsy. Some authors had previously reported on the effectiveness of LF-rTMS over the orbitofrontal cortex in treating neuropsychiatric disorders such as major depression [26] or OCD [27]. Several lines of evidence have shown that the OFC has connections with multiple neural areas such as the hypothalamus, the amygdala, and the somatosensory cortex that are involved in emotional function [28]. The amygdala, for example, receives inputs from the OFC and has been shown to have a functional relationship with the OFC according to some rat studies [28]. As was already mentioned before, the bilateral OFC was chosen as a target due to its accessibility as well as due to its significant connectivity with these neural areas and finally because of the bilateral nature of the patient's neuronal migration disorder. These results take us a step further in personalizing and adapting rTMS targets to patients' individual symptoms rather than categorical diagnoses in the years to come.

\section{Conclusion}

We believe that bilateral OFC rTMS treatment can be well tolerated in patients with frontal epilepsy although the findings of the present case report with regard to clinical efficacy warrant further investigation. Future placebo-controlled, double-blinded randomized controlled trials with sufficiently powered sample sizes are needed to conclusively determine the clinical value of target-specific rTMS in the treatment of epilepsy.

\section{Statement of Ethics}

The research was conducted ethically in accordance with the World Medical Association Declaration of Helsinki. As this was a single retrospective case report, we did not seek a bioethics approval; however, a relevant informed consent was obtained. The relevant consent for publication could be provided upon request. The subject in this study gave written informed consent for publication of her case including publication of images.

\section{Conflict of Interest Statement}

The authors have no conflicts of interest to declare.

\section{Funding Sources}

The authors received no specific funding for this work.

\section{Author Contributions}

All authors agree with the contents of the manuscript and were fully involved in the study and preparation of the manuscript. All authors have read the final version of the manuscript and have approved the submission. Georgios Mikellides, Panayiota Michael, and Angelos 
Gregoriou drafted the manuscript. Teresa Schuhmann and Alexander T. Sack helped to draft the manuscript and supervised the project.

\section{Data Availability Statement}

All data generated or analyzed during this study are included in this article. Further enquiries can be directed to the corresponding author.

\section{References}

1 World Health Organization. Epilepsy [Internet]. 2019 [cited 2019 Jun 20]. Available from: https://www.who. int/news-room/fact-sheets/detail/epilepsy.

2 Fisher RS, van Emde Boas W, Blume W, Elger C, Genton P, Lee P, et al. Epileptic seizures and epilepsy: definitions proposed by the International League Against Epilepsy (ILAE) and the International Bureau for Epilepsy (IBE). Epilepsia. 2005 Apr;46(4):470-2.

3 López González FJ, Rodríguez Osorio X, Gil-Nagel Rein A, Carreño Martínez M, Serratosa Fernández J, Villanueva Haba V, et al. Drug-resistant epilepsy: definition and treatment alternatives. Neurologia. 2015 Sep;30(7): $439-46$.

4 Sultana B, Panzini MA, Veilleux Carpentier A, Comtois J, Rioux B, Gore G, et al. Incidence and prevalence of drug-resistant epilepsy: a systematic review and meta-analysis. Neurology. 2021 Apr 27;96(17):805-17.

5 Melvin JJ, Huntley Hardison H. Immunomodulatory treatments in epilepsy. Semin Pediatr Neurol. 2014 Sep: 21(3):232-7.

6 Sampaio LP. Ketogenic diet for epilepsy treatment. Arq Neuropsiquiatr. 2016 Oct; 74(10):842-8.

7 Téllez-Zenteno JF, Dhar R, Wiebe S. Long-term seizure outcomes following epilepsy surgery: a systematic review and meta-analysis. Brain. 2005 May;128(Pt 5):1188-98.

8 Salanova V, Witt T, Worth R, Henry TR, Gross RE, Nazzaro JM, et al. Long-term efficacy and safety of thalamic stimulation for drug-resistant partial epilepsy. Neurology. 2015 Mar 10;84(10):1017-25.

9 Krishna V, Sammartino F, King NK, So RQ, Wennberg R. Neuromodulation for epilepsy. Neurosurg Clin N Am. 2016 Jan;27(1):123-31.

10 Berényi A, Belluscio M, Mao D, Buzsáki G. Closed-loop control of epilepsy by transcranial electrical stimulation. Science. 2012 Aug 10;337(6095):735-7.

11 Yang D, Wang Q, Xu C, Fang F, Fan J, Li L, et al. Transcranial direct current stimulation reduces seizure frequency in patients with refractory focal epilepsy: a randomized, double-blind, sham-controlled, and three-arm parallel multicenter study. Brain Stimul. 2020 Jan-Feb;13(1):109-16.

12 Fregni F, Otachi PT, Do Valle A, Boggio PS, Thut G, Rigonatti SP, et al. A randomized clinical trial of repetitive transcranial magnetic stimulation in patients with refractory epilepsy. Ann Neurol. 2006 Oct;60(4):447-55.

13 Klomjai W, Katz R, Lackmy-Vallée A. Basic principles of transcranial magnetic stimulation (TMS) and repetitive TMS (rTMS). Ann Phys Rehabil Med. 2015 Sep;58(4):208-13.

14 Lefaucheur JP, Aleman A, Baeken C, Benninger DH, Brunelin J, Di Lazzaro V, et al. Evidence-based guidelines on the therapeutic use of repetitive transcranial magnetic stimulation (rTMS): an update (2014-2018). Clin Neurophysiol. 2020 Feb;131(2):474-528.

15 Rossi S, Antal A, Bestmann S, Bikson M, Brewer C, Brockmöller J, et al. Safety and recommendations for TMS use in healthy subjects and patient populations, with updates on training, ethical and regulatory issues: expert guidelines. Clin Neurophysiol. 2021 Jan;132(1):269-306.

16 Lerner AJ, Wassermann EM, Tamir DI. Seizures from transcranial magnetic stimulation 2012-2016: results of a survey of active laboratories and clinics. Clin Neurophysiol. 2019 Aug;130(8):1409-16.

17 Rossi S, Hallett M, Rossini PM, Pascual-Leone A, Safety of TMS Consensus Group. Safety, ethical considerations, and application guidelines for the use of transcranial magnetic stimulation in clinical practice and research. Clin Neurophysiol. 2009 Dec;120(12):2008-39.

18 Mishra A, Maiti R, Mishra BR, Jena M, Srinivasan A. Effect of repetitive transcranial magnetic stimulation on seizure frequency and epileptiform discharges in drug-resistant epilepsy: a meta-analysis. J Clin Neurol. 2020 Jan;16(1):9-18.

19 Sun W, Mao W, Meng X, Wang D, Qiao L, Tao W, et al. Low-frequency repetitive transcranial magnetic stimulation for the treatment of refractory partial epilepsy: a controlled clinical study. Epilepsia. 2012 Oct;53(10): $1782-9$.

20 Borckardt JJ, Nahas Z, Koola J, George MS. Estimating resting motor thresholds in transcranial magnetic stimulation research and practice: a computer simulation evaluation of best methods. J ECT. 2006 Sep;22(3): $169-75$.

21 Liu WZ, Zhang WH, Zheng ZH, Zou JX, Liu XX, Huang SH, et al. Identification of a prefrontal cortex-to-amygdala pathway for chronic stress-induced anxiety. Nat Commun. 2020 May 6;11(1):2221. 
22 Liang X, Zebrowitz LA, Aharon I. Effective connectivity between amygdala and orbitofrontal cortex differentiates the perception of facial expressions. Soc Neurosci. 2009;4(2):185-96.

23 Menkes DL, Gruenthal M. Slow-frequency repetitive transcranial magnetic stimulation in a patient with focal cortical dysplasia. Epilepsia. 2000 Feb;41(2):240-2.

24 Tergau F, Naumann U, Paulus W, Steinhoff BJ. Low-frequency repetitive transcranial magnetic stimulation improves intractable epilepsy. Lancet. 1999 Jun 26;353(9171):2209.

25 Mantovani A, Lisanby SH, Pieraccini F, Ulivelli M, Castrogiovanni P, Rossi S. Repetitive Transcranial Magnetic Stimulation (rTMS) in the treatment of panic disorder (PD) with comorbid major depression. J Affect Disord. 2007 Sep;102(1-3):277-80.

26 Feffer K, Fettes P, Giacobbe P, Daskalakis ZJ, Blumberger DM, Downar J. 1Hz rTMS of the right orbitofrontal cortex for major depression: safety, tolerability and clinical outcomes. Eur Neuropsychopharmacol. 2018 Jan; 28(1):109-17.

27 Nauczyciel C, Le Jeune F, Naudet F, Douabin S, Esquevin A, Vérin M, et al. Repetitive transcranial magnetic stimulation over the orbitofrontal cortex for obsessive-compulsive disorder: a double-blind, crossover study. Transl PsychiatryD. 2014 Sep 9;4(9):e436.

28 Rempel-Clower NL. Role of orbitofrontal cortex connections in emotion. Ann N Y Acad Sci. 2007 Dec;1121: 72-86.

29 Theodore WH, Hunter K, Chen R, Vega-Bermudez F, Boroojerdi B, Reeves-Tyer P, et al. Transcranial magnetic stimulation for the treatment of seizures: a controlled study. Neurology. 2002 Aug 27;59(4):560-2.

30 Fregni F, Thome-Souza S, Bermpohl F, Marcolin MA, Herzog A, Pascual-Leone A, et al. Antiepileptic effects of repetitive transcranial magnetic stimulation in patients with cortical malformations: an EEG and clinical study. Stereotact Funct Neurosurg. 2005;83(2-3):57-62.

31 Santiago-Rodríguez E, Cárdenas-Morales L, Harmony T, Fernández-Bouzas A, Porras-Kattz E, Hernández A. Repetitive transcranial magnetic stimulation decreases the number of seizures in patients with focal neocortical epilepsy. Seizure. 2008 Dec;17(8):677-83. 